\title{
Optimal Rate Control in Wireless Networks with Fading Channels
}

\author{
Javad Razavilar, ${ }^{1}$ K.J. Ray Liu, ${ }^{2}$ and Steven I. Marcus ${ }^{2}$ \\ ${ }^{1} 3 \mathrm{COM}$ Labs, 3COM Inc. \\ 12230 World Trade Drive \\ San Diego, CA 92128 \\ javad_razavilar@3com.com \\ ${ }^{2}$ Electrical Engineering Department and Institute for Systems Research \\ University of Maryland \\ College Park, MD 20742
}

\begin{abstract}
A dynamic programming optimization method is used to obtain the optimal rate control policy in a wireless network with fading channel. In a wireless network it is assumed that the base station is capable of transceiving data packets at two rates, either $R_{h}$ or $R_{l}\left(R_{h}>R_{l}\right)$. An optimal policy is derived which jointly minimizes the transmission delay and the number of rate switchings in the network. Numerical results indicate that by sacrificing only $1 \%$ of transmission quality in terms of the average delay one can achieve more than $50 \%$ reduction in switching load of the network. Our analytical as well as numerical results confirm that the optimal policy is a threshold policy.
\end{abstract}

Keywords: Optimal Rate Control, Dynamic Programming, Wireless Networks.

\section{Introduction}

The increasing popularity of the wireless network services with limited amount of available resources calls for highly efficient resource allocation methods [1],[2], [3]. One of the major issues in wireless data networks is the rate allocation (or control) problem. This is especially important in the downlink, since in a wireless data network most of the traffic flow is from the base station to mobiles, e.g. an Internet connection or a multimedia (voice/image/data) connection. In this paper, we investigate the rate control problem for wireless channels from an optimal control point of view. There exists some literature on obtaining the nature of optimal control policies for a wide range of related problems [1],[3],[4]. In [2], the authors consider the problem of stochastic control of handoffs in a cellular networks and formulate an optimal policy for base station handoff problem.
In this paper, we derive some properties of a class of optimal rate control problem using the theory of dynamic programming (DP). The general nature of the problem considered is as follows. In a wireless network, the base station is capable of transmitting data packets at two rates, either $R_{h}$ or $R_{l}\left(R_{h}>R_{l}\right)$. These two rates could correspond to two different modulation schemes such as 32-ary PSK and QPSK. The base station transmits the data packets over a wireless channel to mobile users. The received SNR by the mobile users is subject to fluctuation due to fading and noise. We assume a finite state Markov model (FSM) for the wireless channel. The mobile constantly monitors the received signal to noise ratio (SNR). At each measurement instant the mobile observes the state of the channel and determines which state of channel it belongs to. At each decision making instant by employing an optimal strategy, mobile decides whether to send a request to the base station to switch the rate or not. For this purpose there is a feedback channel (assumed to be noise free) so the user can send its request to the base station. Figure 1 illustrates the block diagram of a system where the mobile employs an optimal strategy in choosing the rate in the network.

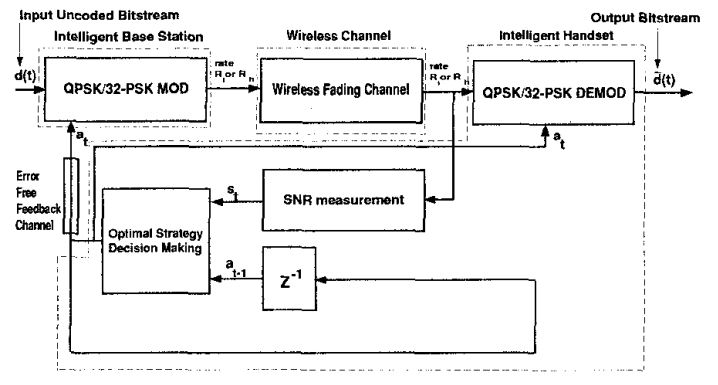

Figure 1: Block diagram of a system where the mobile employs an optimal strategy in chossing the data rate in the network. 
The optimal policy which determines the choice of rates (or modulation schemes) jointly minimizes the delay in sending the packets and the number of rate switchings. We show that under certain conditions the optimal strategy has the form of a threshold policy. In a wireless network, shadowing and fading effects result in signal strength variations in mobile environment. This may cause unnecessary and frequent rate switchings which is highly undesirable, because it translates to protocol overheads to switch the rate (rate negotiation phase). An improperly designed rate control algorithm can result in an unacceptably high level of bouncing (resulting in high signaling costs) and/or a high probability of forced termination. Delaying a rate switching as the signal strength received from the base station starts to deteriorate may result in lost data transmission session. A good rate control algorithm also reduces the occurrence of the involunteer termination of the data transmission in the network.

The paper is organized as follows. Section 2 reviews some of the relevant results from the theory of dynamic programming. In Section 3 a finite state Markov channel model for wireless Rayleigh fading channels is presented. Optimal data rate control problem cast as an infinite horizon discounted cost dynamic programming problem forms the subject of Section 4. The average delay of transmitting the packets and expected number of rate switchings are discussed in Section 5. Numerical results are presented in Section 6. Section 7 includes our conclusions and remarks.

\section{Dynamic Programming}

In this section, we review some of the relevant results from the theory of dynamic programming [5],[6],[7]. which will be used subsequently to derive the nature of optimal policies for a class of rate control problems. The stochastic model of the wireless channel is such that the states of the underlying Markov model of the channel evolves according to a time invariant Markov transition rule independent of past and present actions (chosen rate) taken by the mobile. Let $\left\{s_{t}\right\}_{t=0}^{\infty}$ be a discrete time process. At any given time the state of the channel $s_{t}$ takes its value from a countable state space denoted by the set of non-negative integers $\{0,1,2, \ldots, K-1\}$. In our problem this set represents the finite state space of the underlying Markov model of the channel. At each time instant $t \in\{0,1,2 \ldots\}$, we are required to choose an "action" $a_{t}, a_{t} \in A$, where $A$ denotes the given set of all admissible actions. In our rate control problem the set of admissible actions is $A=\left\{R_{l}, R_{h}\right\}$ and the action is to choose one of these two rates. From now on we encode the set of admissible actions with 0 for $R_{l}$ and 1 for $R_{h}$, therefore $a_{t} \in\{0,1\}$. Let us assume that the optimal action (chosen rate) taken by the mobile for the time slot $[t, t+1)$ is denoted by $a_{t}$. Therefore, $a_{t-1}$ denotes the optimal action taken by the mobile for the previous time slot $[t-1, t)$. Now let us define the aggregate state of the system as $\left(s_{t}, a_{t-1}\right)$ which takes values in $\{0,1, \cdots, K-1\} \times\{0,1\}$. Suppose that for time slot $[t, t+1)$ the mobile chooses the optimal action (rate) $a_{t}$ while the aggregate state of the system is $\left(s_{t}, a_{t-1}\right)$. Then we incur an instantaneous cost $R\left(s_{t}, a_{t-1}, a_{t}\right)$, which is a mapping from the finite space $R:\{0,1,2 \ldots, K-1\} \times\{0,1\} \times\{0,1\} \mapsto \mathbb{R}$, where $\mathbb{R}$ denotes the set of real numbers. The optimal policy $\pi$ is a mapping from the aggregate state space to the action space, i.e. $\pi:\{0,1,2 \ldots, K-1\} \times\{0,1\} \mapsto\{0,1\}$. Given the evolution of the aggregate state of the system $\left\{s_{t}, a_{t-1}\right\}_{t=0}^{\infty}$, we are interested in the solution of the following problem:

Choose $\left\{a_{t}\right\}_{t=0}^{\infty}$ such that

$$
V^{\pi}(s, i) \triangleq E_{(s, i)}^{\pi}\left[\sum_{t=0}^{\infty} \beta^{t} R\left(s_{t}, a_{t-1}, a_{t}\right)\right],
$$

is minimized, where $E_{i}^{\pi}$ denotes the expectation under the policy $\pi$. With $a_{-1}$ being arbitrary, the initial state $s_{0}=i$, and $0<\beta \leq 1$ is the discount factor. This problem is called an infinite horizon discounted cost problem. The above cost reflects the fact that while choosing the action $a_{t}$ at time slot $[t, t+1)$, we would like to take into account the effect of this action on the future behavior of the system.

An important subclass of policies, which are of particular interest, is the class of stationary policies. If the mapping rule $\pi_{t}$ does not depend on time $t$, the mapping is said to be a time-invariant mapping or a stationary policy. If a stationary policy $\pi$ is employed, then the sequence of the states evolved in time forms a Markov chain and the evolution of states in time is called a Markov decision process (MDP) [5],[6],[7].

\section{Markov Model for Wireless Channels}

The multipath effect in a wireless network results in the fluctuation of the received signal envelope that is Rayleigh distributed. The p.d.f. of a random variable distributed according to a Rayleigh distribution is illustrated in Figure 2.

This channel is known as Rayleigh fading channel. Any partition of the received SNR into a finite number of intervals forms a finite state channel model. Let $A_{0}<A_{1}<A_{2} \cdots<A_{K}=\infty$ be the thresholds of the received SNR. Then the Rayleigh fading channel is said to be in state $k, k=0,1, \cdots, K-1$, if the received SNR is in the interval $\left[A_{k}, A_{k+1}\right.$ ) [8]. To calculate the transition probabilities $p_{i j}$ we make the following assumption: $p_{i j}=0,|i-j|>1$. The Markov model for a multipath fading wireless channel is illustrated in Figure 3. 


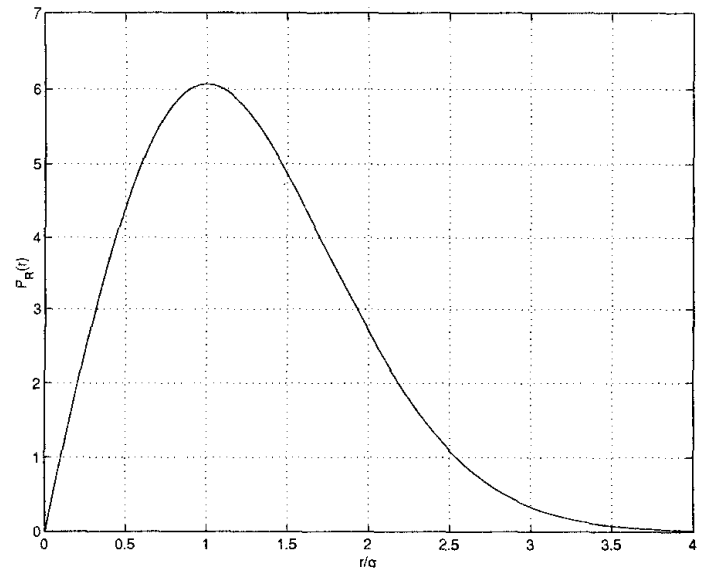

Figure 2: PDF of a random variable distributed according to a Rayleigh distribution.
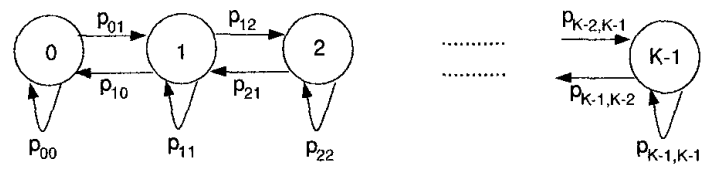

Figure 3: $K$-state noisy channel with Markov transitions modeling a Rayleigh fading Channel.

\section{Optimal Data Rate Control}

In this section we introduce a cost function which captures the desired trade-off between data transmission quality and switching cost, in an appropriate balanced manner for the optimal rate control (allocation) problem. In order to have a reasonable cost-per-stage $R$, each time the mobile unit switches between two rates rate $R_{l}$, and $R_{h}$ this should be penalized by a cost associated with rate switching. Let $C_{s}$ denote the cost of the rate switching. On the other hand a reward $d_{0}$ (or $d_{1}$ ) encourages the mobile unit to switch the rate in order to minimize the transmission delay in the network. Therefore $d_{0}$ and $d_{1}$ should correspond to transmission delay associated with the respective rate (or equivalently respective modulation). Associated with each modulation scheme there is a $P_{e}$ probability of symbol error versus SNR curve. The probability of symbol error for $M$-ary PSK modulation for high SNR is given by $[9]$

$$
P_{e M}\left(\gamma_{s}\right)=2 Q\left(\sqrt{2 \gamma_{s}} \sin \frac{\pi}{M}\right)
$$

Where $\gamma_{s}$ is symbol SNR and $Q($.$) is the Q-function.$ Using the probability of symbol error, $P_{e}$, we can define a quantity which reflects the transmission delay associated with the corresponding modulation scheme. We use first order approximation of transmission delay $d_{0}$ and $d_{1}$ as follows

$$
\begin{aligned}
& d_{1}\left(s_{t}\right)=\frac{G}{k_{1}\left(1-P_{e 1}\left(s_{t}\right)\right)} \\
& d_{0}\left(s_{t}\right)=\frac{G}{k_{0}\left(1-P_{e 0}\left(s_{t}\right)\right)}
\end{aligned}
$$

where $G$ is a constant, $k_{1}\left(k_{0}\right)$ is the number of bits per symbol and $P_{e 1}\left(P_{e 0}\right)$ is the symbol error probability for rate $R_{h}\left(R_{l}\right)$. In Figure 4 , these two functions $d_{1}$ and $d_{0}$ are plotted for 32-ary PSK and QPSK modulations.

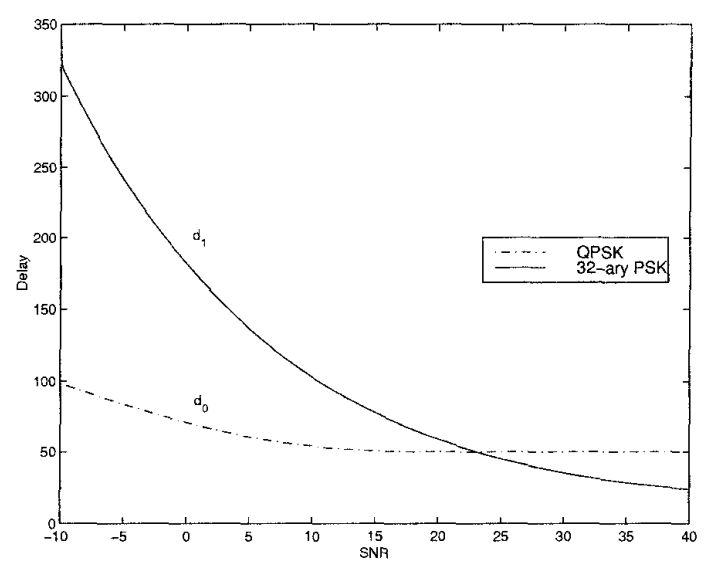

Figure 4: A function of transmission delay for 32-ary PSK and QPSK modulations.

The final cost-per-stage function $R$ is defined as follows:

$$
\begin{aligned}
& R\left(s_{t}, a_{t-1}, a_{t}\right) \\
& \quad= \begin{cases}C_{s} & a_{t-1} \neq a_{t} \\
(-1)^{a_{t-1}}\left(d_{0}\left(s_{t}\right)-d_{1}\left(s_{t}\right)\right) & a_{t-1}=a_{t}\end{cases}
\end{aligned}
$$

Now the problem at hand is to solve the following minimization problem

$$
V(s, i)=\min _{u=0,1} E_{s, i}^{\pi}\left[\sum_{t=0}^{\infty} \beta^{t} R\left(s_{t}, a_{t-1}, a_{t}\right)\right]
$$

for every $(s, i)$ in $\{0,1,2, \ldots, K-1\} \times\{0,1\}$ and policy $\pi$. Now using the state transition probabilities $p_{i j}$ we define the following quantity

$$
\tilde{V}_{n-1}(s, i)=\sum_{s^{\prime}} p_{s s^{\prime}} V_{n-1}\left(s^{\prime}, i\right)
$$

Then the DP equation for the problem at hand is simply

$$
\begin{aligned}
& V_{n}(s, i) \\
& \quad=\min _{u=0,1}\left\{R(s, i, u)+\beta \tilde{V}_{n-1}(s, u)\right\}
\end{aligned}
$$


or equivalently,

$$
\begin{aligned}
V_{n}(s, i) & \\
=\min & \left\{C_{s}+\beta \tilde{V}_{n-1}(s, i \oplus 1),\right. \\
& \left.(-1)^{i}\left(d_{0}(s)-d_{1}(s)\right)+\beta \tilde{V}_{n-1}(s, i)\right\}
\end{aligned}
$$

where $\oplus$ denotes modulo two addition. Moreover, the optimal policy $\pi^{\star}$ is a Markov stationary policy which selects to switch in state $(s, i)$ if and only if

$C+\beta \tilde{V}_{n-1}(s, i \oplus 1) \leq(-1)^{i}\left(d_{0}(s)-d_{1}(s)\right)+\beta \tilde{V}_{n-1}(s, i)$

An important observation regarding the solution of the discounted DP problem given by (7) is that it can be interpreted as the fixed point of a well defined operator such as $T$ where $T V=V$. Motivated by the form of the dynamic programming equation (7), we associate $R$-valued mappings $\tilde{T} \varphi$ and $T_{u} \varphi, u=0,1$ defined on $\{0,1,2, \ldots, K-1\} \times\{0,1\}$ by setting

$$
(\tilde{T} \varphi)(s, i)=\sum_{s^{\prime}} p_{s s^{\prime}} \varphi\left(s^{\prime}, i\right)
$$

and

$$
\left(T_{u} \varphi\right)(s, i)=R(s, i, u)+\beta(\tilde{T} \varphi)(s, u)
$$

for $(s, i) \in\{0,1,2, \ldots, K-1\} \times\{0,1\}$. Next, we introduce the operator $T$ by setting

$$
(T \varphi)(s, i)=\min _{u=0,1}\left(T_{u} \varphi\right)(s, i)
$$

for every $\varphi$. This operator permits a rewriting of the dynamic programming equation as $V=T V$, so that $V$ is identified as the unique fixed point of the operator $T$.

A rate switching policy $\pi$ is said to be a threshold policy with threshold functions $\tau_{i} i=0,1$, if it is a Markov stationary policy such that

$$
\pi^{\star}(s, 0)=1 \quad \text { iff } \quad z(s) \geq \tau_{0},
$$

and

$$
\pi^{\star}(s, 1)=0 \quad \text { iff } \quad z(s) \leq \tau_{1}
$$

where $z(s)=d_{0}(s)-d_{1}(s)$, for every $s \in\{0,1,2, \ldots, K-$ $1\}$.

Proposition 1: Under the model assumptions, the optimal rate allocation (control) policy $\pi^{\star}$ is a threshold policy with threshold functions $\tau_{i}^{\star}:\{0,1,2, \ldots, K-$ $1\} \rightarrow R, i=0,1$, which are uniquely determined and $\tau_{1}^{\star}<\tau_{0}^{\star}$ for all $s$ in $\{0,1,2, \ldots, K-1\}$.

Proof: Please refer to [3].

\section{Average Delay and Rate Handoffs}

Once a rate control (allocation) policy (be it optimal or not) has been selected, it is of interest to compute the average delay of transmitting the packets over the wireless channel and the expected number of rate switchings that the mobile experiences while the optimal policy is in effect. These two quantities constitute good measures of the effectiveness of a rate control policy.

We define the average delay $D_{\pi}$ of the policy $\pi$ to be the the mean value of the delay of the selected rate to receive the packets from the base station under the policy $\pi$ during the packet transmission, namely

$$
D_{\pi}(s, i)=\mathbf{E}_{s, i}^{\pi}\left[\sum_{t=0}^{\infty} \beta^{t}\left(I_{t} d_{1}\left(s_{t}\right)+\left(1-I_{t}\right) d_{0}\left(s_{t}\right)\right]\right.
$$

where $I_{t}$ is a Bernoulli random variable with $I_{t} \in\{0,1\}$, and $\operatorname{Pr}\left(I_{t}=1\right)=1-\operatorname{Pr}\left(I_{t}=0\right)=\operatorname{Pr}\left(\pi_{t}=1\right)$. On the other hand, the expected number of rate switchings under the policy $\pi$ is defined by

$$
S_{\pi}(s, i)=\mathbf{E}_{s, i}^{\pi}\left[\sum_{t=0}^{\infty} \beta^{t} \mathbf{1}\left[a_{t-1} \neq a_{t}\right]\right]
$$

where $\mathbf{1}($.$) is the indicator function. Both D_{\pi}$ and $S_{\pi}$ can be written as discounted cost functions. For any Markov stationary policy $\pi$, and in particular for any threshold policy, this fact can be exploited for numerical purposes by interpreting $D_{\pi}$ and $S_{\pi}$ as fixed points for suitably defined contraction mappings.

\section{Numerical Results}

In this section, we use numerical methods to find the solution to the optimization problem posed in (5). It is demonstrated that the optimal policy is indeed a threshold policy. This corroborates the results of Proposition 1.

In our simulations, $R_{l}$ corresponds to a QPSK modulation and $R_{h}$ corresponds to 32-ary PSK modulation. The numerical techniques are employed to find the optimal policy for two cases, $C_{s}=0$, and $C_{s}=45$. Figure 5 illustrates how the rate switching $\operatorname{cost} C_{s}$ affects the optimal thresholds $\tau_{0}$ and $\tau_{1}$. These optimal thresholds along with the transmission delay curves, $d_{0}$ and $d_{1}$, are plotted all in the same figure for comparison purposes. The effectiveness of the optimal policy is assessed by comparing the average delay $D_{\pi}$ and expected number of rate switchings $S_{\pi}$ for different values of the switching cost $C_{s}$. Figure 6 illustrates how $D_{\pi}$ and $S_{\pi}$ behave while switching $\operatorname{cost} C_{s}$ varies.

\section{Conclusions}

In this chapter we studied the problem of optimal rate control in wireless networks. A stochastic optimization 

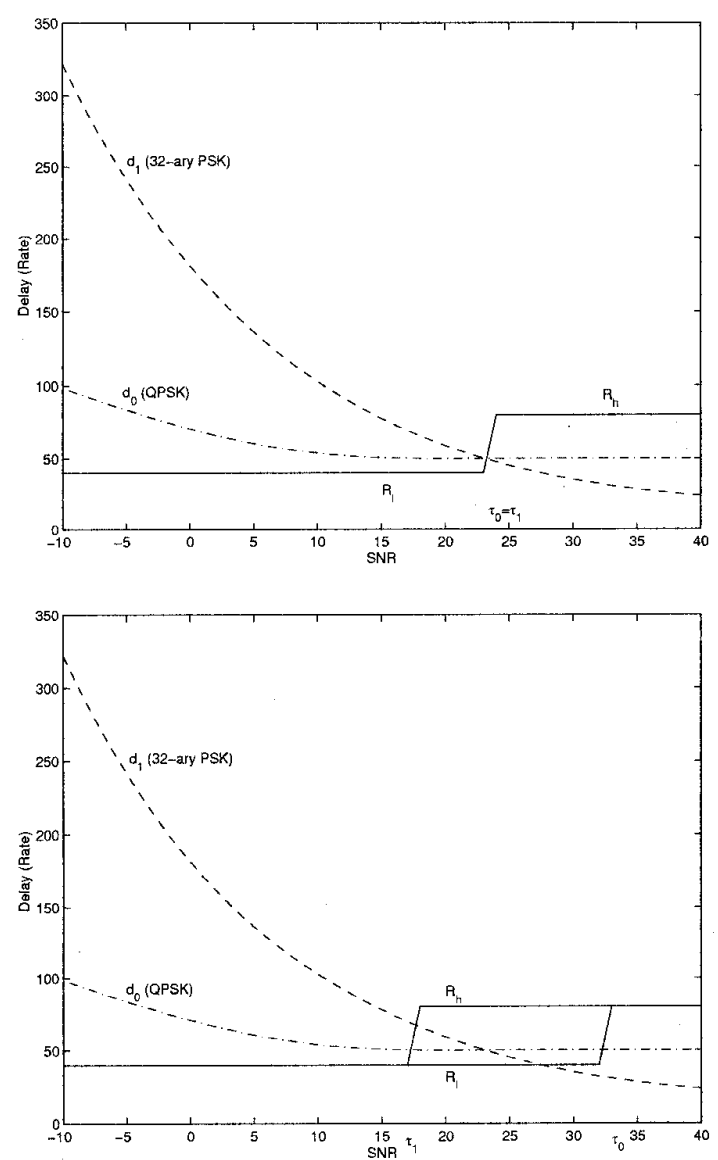

Figure 5: Optimal thresholds $\tau_{0}$ and $\tau_{1}$ with $C_{s}=0$ (top), $C_{s}=45$ for switching between $R_{h}$ and $R_{l}$ over a wireless Rayleigh fading channel.

technique based on dynamic programming method is used to obtain the optimal policy. Using the results from the theory of dynamic programming, it is shown that, the optimal policy for rate control problem is in the form of a threshold policy - a property of significance interests both from the analytical and implementation points of view.

\section{References}

[1] A. Ephremides and S. Verdu, "Control and optimization methods in communication network problems," IEEE. Trans. Automatic Control, vol. 34, pp. 930-942, September 1989.

[2] R. Rezaiifar, A. M. Makowski, and S. Kumar, "Stochastic control of handoffs in cellular networks," IEEE J. Selected Areas in Communications, vol. 13, Sept. 1995.

[3] J. Razavilar, Signal Procesing and Performance Analysis for Optimal Resource Allocation in Wireless
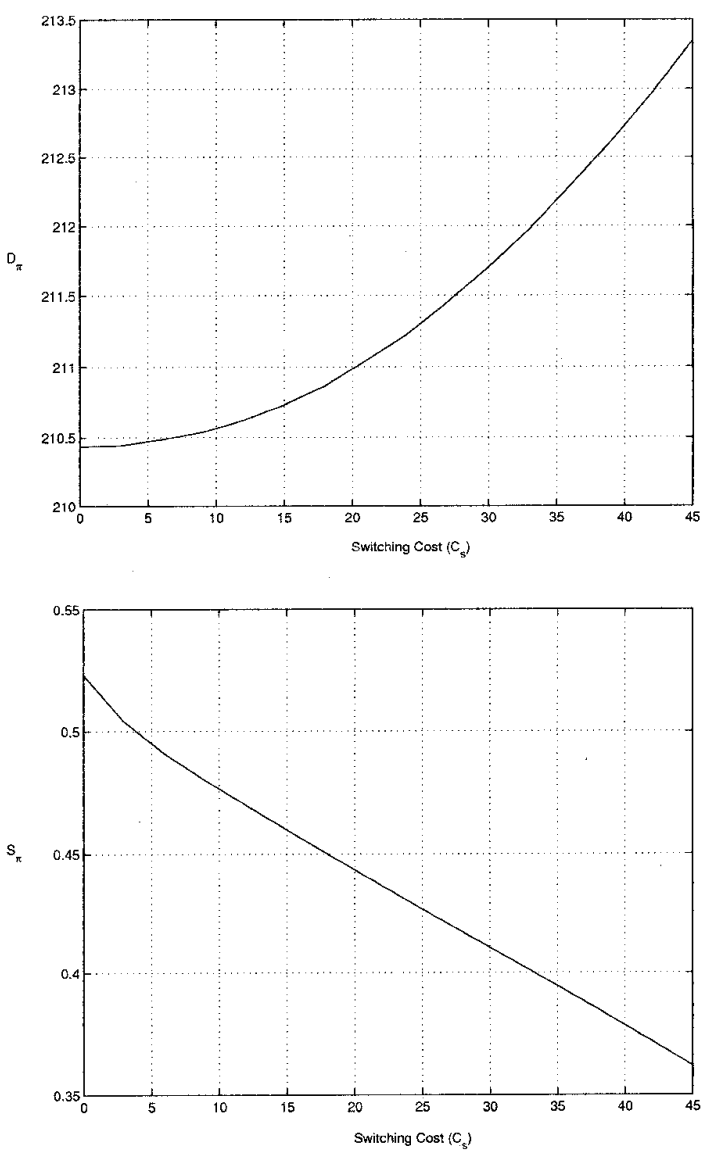

Figure 6: Average delay $D_{\pi}$ (top), average rate switching $S_{\pi}$ versus rate switching cost $C_{s}$.

Networks. PhD thesis, University of Maryland, 1998.

[4] N. Yin and M. G. Hluchyj, "A dynamic rate control mechanism for source coded traffic in a fast packet network," IEEE J. Selected Areas in Communications, vol. 9, pp. 1003-1012, Sept. 1991.

[5] M. L. Puterman, Markov Decision Processes, Discrete Stochastic Dynamic Programming. John Wiley, 1994.

[6] D. P. Bertsekas, Dynamic Programming and Optimal Control. Athena Scientific, 1995.

[7] S. M. Ross, Stochastic Dynamic Programmin. Academic Press, 1983.

[8] H. S. Wang and N. Moayeri, "Finite-state markov channel-a useful model for radio communication channels," IEEE Trans. on Vehicular Technology, vol. 44, pp. 163-170, Feb. 1995.

[9] J. G. Proakis, Digital Communications. New York: McGraw Hill, third ed., 1995. 